\title{
The 20th International Reid Bioanalytical Forum
}

\section{The 20th International Reid Bioanalytical Forum \\ University of Surrey, Guildford, UK, 9-12 September 2013}

The 20th International Reid Bioanalytical Forum was attended by a little over 100 participants from pharma, contract research, instrument vendors and academia. The Forum was divided into six broad themes, which included collaborative partnerships between $\mathrm{CROs}$ and pharmaceutical companies, technology developments, problem solving, the bioanalytical toolbox, biomarker analysis and regulations in bioanalysis.

\section{Overview}

The Bioanalytical Forum, initiated by Dr Eric Reid in 1975, is held every two years at the University of Surrey (Surrey, UK). The Forum is organized by the Forum Syndicate under the auspices of the Chromatographic Society following an initial donation from Reid. In recognition of the contribution of Reid, who sadly he passed away in 2010, the series has been renamed 'The International Reid Bioanalytical Forum' [1].

The Chromatographic Society itself is an international organization devoted to the promotion and dissemination of knowledge on all aspects of chromatography and related separation techniques. The Society was founded in 1956 and runs a number of 1 day and longer short courses and symposia. The Forum Syndicate is a subcommittee of the Chromatographic Society specifically drawn together to organize the International Reid Bioanalytical Forum; its members are currently Howard Hill, Ian Wilson (chairman and co-organizer), Derek Stevenson, Forum (co-organizer), Stephen Westwood, John Smeraglia, Ray Briggs, Richard Houghton and Timothy Sangster.

The Forum followed a familiar format for regular participants, although the timing had been moved in the conference calendar from the usual July to a September date. The meeting commenced after lunch on Monday (9 September 2013) and ended at lunchtime on the Thursday. Encouragingly, there was no drop-off in attendance on the final morning session which covered regulatory aspects, including an excellent talk from Christine Gray (MHRA, London, UK) and a presentation uncovering the practicalities of capillary micro-sampling. The conference included two separate exhibitions; Tuesday for instrument manufacturers and Wednesday populated largely by CROs.
Over 20 posters were on permanent display in the exhibition hall for delegates to gather ideas when not networking or enjoying the excellent refreshments.

The social events followed a tried and tested format. There was a buffet meal and drinks reception in the Wates House bar on the first evening, the Tuesday evening event was an off-campus dinner at Clandon House, a local National Trust property, and Wednesday was the conference dinner in the university Hillside restaurant. The organizers would like to thank all exhibitors and sponsors of the event, especially AB SCIEX for sponsoring the conference dinner and drinks, Biotage for their support of the Wates House reception and Future Science for advertising the event in Bioanalysis.

\section{Collaborative partnership between CROs \& pharmaceutical companies}

The conference was initiated with the customary welcome, introduction and house-keeping address by Stevenson. This followed straight into the first session, chaired by Smeraglia, on the theme of 'Collaborative partnership between CROs and pharmaceutical companies', and specifically a presentation by Neil Spooner (GSK, Middlesex, UK) on building positive relationships with CROs. Part of Spooner's role at GSK is optimizing the interfaces with CROs that support nonclinical bioanalytical work. He described the current trends and drivers on pharmaceutical companies to outsource increasing amounts of their bioanalytical and TK work. This shift to the generation of critical study data outside the innovator, often with reduced in-house bioanalytical expertise and capability, in turn leads to an increasing need for cooperation and trust between both sponsor and service provider. GSK outsourcing of PK bioanalysis has

\section{Richard Houghton}

Quotient Bio Analytical Sciences, Newmarket Road, Fordham, Cambridge, CB7 5WW, UK E-mail: richard.houghton@quotient bioresearch.com 
grown from just over 30\% in September 2010 to very nearly $75 \%$ in March 2012, while TK outsourcing has risen from approximately 11 to $28 \%$ in the same period. The push to outsource more bioanalysis has resulted in greater flexibility of staff resource, tighter control of overhead costs, more transparent costs to project teams and a refocusing of internal resource. Spooner went on to describe how GSK are looking to develop collaborations with service providers but concluded that the key attribute to success were quality, communication and trust.

The pharmaceutical industry is experiencing what is, without doubt, the most turbulent time in its history. Drug-development costs have escalated; fewer molecules have gained regulatory approval over recent years, while at the same time the larger companies have suffered the impact of the patent-cliff. Christine Robinson (HLS, Cambridgeshire, UK) summarized the challenges facing the industry and reviewed strategies that companies are adopting; diversification, consolidation, partnering, emerging markets, precompetitive data sharing and 'fail faster' science. Robinson moved on to consider what the industry of the future may look like and the roles and responsibilities of academia, biotech, large pharma and the CRO industries in developing new medicines. However, she concluded a thought-provoking talk that all is not lost; US FDA approvals increased in 2012, there is a greater focus in the industry on biological treatments with reduced financial patent cliffs and opportunities for personalized medicine and the development of companion diagnostics.

Graeme Clark (Cyprotex, Cheshire, UK) followed with a talk on exploratory bioanalysis and the risks associated of outsourcing this type of work with traditionally no structural information provided with the molecule. He illustrated this with a real example of a molecule, which had been supplied with the potential to be transformed into an acyl-glucuronide metabolite. If structural information had been provided along with the molecule, the bioanalytical risks would have been identified earlier in this particular case. The final talk of this first session was from Richard Hucker (A4P Consulting Ltd, Kent, UK) who reinforced some of the ideas presented earlier in the session on the changing face of bioanalysis. He emphasized the increasing size and complexity of clinical trials and use of safety and efficacy biomarkers earlier in development that is, first in human trials.

After a short break, the session resumed with a presentation from Suzy Rigby (consultant,
UK) who continued on the theme of new models of working for more effective management of evolving challenges and relationships. This was followed by Graeme Smith (HLS) who took a slightly different tack and described the use of low-tech, cost-effective tools such as Six Sigma and LEAN processes for improving productivity and quality in bioanalysis. From process mapping, procedures can then be optimized to maximize value-added time, minimize setup tasks and eliminate waste; clearly important as the CRO sector comes under increasing cost pressures.

Wilson was next up to the podium and spoke on the theme of 'smaller, better, faster'. He described the analysis of micro-samples from chimeric and genetically modified mice for drugs and metabolites and the application of this approach to drug-drug interaction studies. Chimeric mice are bred to contain up to $95 \%$ of human hepatocytes; allowing a more representative metabolite profile to be understood before clinical administration. However, these mice cost US\$5000 each and only live for 2 weeks, so you need to work fast! The last talk of the day was delivered by Iain Love (HLS) who spoke on 'Relationships in bioanalytical discovery outsourcing: a paradigm shift'. He introduced a new concept (to me at least) of 'FTE Burn'. It is a metric of outsourcing efficiency and measured as the relative ratio of man-day output at the service provider to internal resource required to monitor the project at the innovator. Clearly, high output with minimal sponsor oversight is optimal but Iain then went on to discuss what is required of both parties to reach this ideal.

\section{Technology developments \& novel tools for bioanalysis}

The theme of the morning session of the first full day of the conference was 'Technology developments and novel tools for bioanalysis'. Steve Taylor (AB SCIEX, Warrington, UK) shared some recent advances in the identification and quantitation of biopharmaceuticals. He covered intact molecular weight characterization of antibodies, peptide mapping using trypsin digestion, the analysis of antibody-drug conjugates and the identification of sequence variants in recombinant biotherapeutic proteins all using the Triple $\mathrm{TOF}^{\mathrm{TM}}$ 5600+ instrument. John Stobaugh (University of Kansas, KS, USA) then spoke about the determination of the protein 3-nitrotyrosine, a post-translational modification which is linked to inflammation and various disease states; challenging because of its low abundance. The current analytical strategies being pursued 
involve derivatization, selective enrichment followed by extreme UPLC separation on long axial columns operated at 30,000 psi. Diego Rodriguez Cabaleiro, presenting on the behalf of Waters, described a series of systematic troubleshooting protocols and tips and tricks developed in order to complement their generic methodologies for the analysis of peptide molecules. More recently, research has progressed to create robust and generic methodologies for the bioanalysis of more complex recombinant proteins and monoclonal antibodies; requiring additional steps for sample preparation that need to be investigated and optimized including the use of micro fluidics chromatography and accurate MS. Derek Hillbeck (Thermo Scientific, Loughborough, UK) finished up this first session of the day with a talk focused on HPLC of biomolecules. He described a number of different separation modes applicable for large molecules but emphasized that the underlying principle of knowing the physicochemical properties of your target molecule remain the key to success. However, often the perception of acceptable chromatography needs to be adjusted, and certainly systems need to be inert with good connection to ensure the best chromatography is obtained.

Following a short break for coffee, the presentations resumed. The first presenter was Jordan Stobaugh (AbbVie, IL, USA) who spoke about an experimental system with a constant pressure, high temperature approach for one dimensional separations capable of $>30,000 \mathrm{psi}$; extreme UPLC. Using commercially available $25 \mathrm{~cm}$ Waters BEH, $1.9 \mu \mathrm{m}$ columns, peak capacities approaching 1000 are possible. He illustrated this with examples of complex mixture separations from fractionated yeast cell lysate digests. He confirmed during the question and answer session following his talk that the $\mathrm{BEH}$ particles appear to be able to withstand these high pressures. Catarina Horro Pita (Quotient Bio Analytial Sciences, Cambridgeshire, UK) spoke next on the use of derivatization in LC-MS/MS, which can dramatically improve the sensitivity and selectivity of an assay, as well as analyte retention, stability and recovery; the talk was illustrated with some of the successful derivatization protocols used recently. Stuart Hassard (Horiba Scientific, Stanmore, UK) then described deltDOT LFII ${ }^{\circledR}$ technology; a multipixel capillary electrophoresis detector. It offers fast, accurate, repeatable data of higher resolution over conventional single point detectors. $\mathrm{He}$ illustrated these advantages with a number of applications including examples of raw material testing, production monitoring, purification, formulation and batch release for biomolecules.

\section{Problem solving}

The theme of the afternoon session was 'Problem solving' with a particular focus on case studies related to bioanalytical challenges. However, first up was the hastily rearranged talk from Zoltan Takats (Imperial College, London, UK), on qualitative tumor profiling during surgery using the iKnife ${ }^{\mathrm{TM}}$. Using an adapted DESI ion source interfaced to a Waters triple quadrupole instrument, he described how it is possible to measure phospholipid/fatty acid distributions which can then be correlated to histological properties of tissue. By measuring real-time patterns of target analytes and applying multivariate statistics, abnormal tissue can be differentiated from healthy tissue during surgery. This allows the surgeon to test and remove only what is necessary increasing positive patient outcome from major invasive surgery; a memorable and very exciting talk. Next was Jean-Pierre Chervet (Antec, The Netherlands) with a presentation on the use of on-line coupling of electrochemistry with MS. This technique has shown great potential in mimicking nature's REDOX reactions in several application areas, such as drug metabolism, protein chemistry, electrochemical synthesis of metabolites, and environmental degradation amongst others. In all these applications the electrochemical cell acts as a reactor in which a controlled oxidation or reduction (REDOX reaction) takes place and the MS is used as detector. This biomimetic oxidation results in significant time and cost saving compared with days or weeks using the traditional in vitro (e.g., microsomes) and/or in vivo methods (e.g., rodents, human, etc.). The remainder of the session was made up of a short audience participation presentation by David Neville (Quotient Bio Analytial Sciences), who illustrated the use of internal standard response as a troubleshooting tool in bioanalytical methods, Elia Hall (YBS, UK) speaking on 'A voyage of discovery: overcoming challenges in highthroughput bioanalysis', Westwood (Thermo Scientific) on 'Advances in sample preparation' and Dan Chapman (Waters, St Albans, UK) on 'Accepting new technology whilst managing the regulatory burden - a vendor's role'.

After a short break, the theme of 'Problem solving' continued with three further talks but now with an emphasis on tissue analysis case studies. Chris Kemper (Pharma Navigators, NJ, USA) offered practical advice on 'Best practices 
in tissue preparation for bioanalysis' and how to collect tissue samples in the laboratory. He also introduced the topic of quantitative tissue imaging using MALDI [2], a prelude to the talk, which was followed by Bianca Squillaci (GSK) specifically on this subject. MALDI imaging MS provides a powerful alternative to whole body autoradiography or LC-MS analysis of tissue homogenate, allowing the direct detection and identification of endogenous (e.g., lipids, proteins and peptides) and exogenous (e.g., drug and metabolites) components in a tissue slice while preserving the spatial distribution. However, it is the combination of MALDI MS imaging data with additional information from pathologists, toxicologists and others that is the real strength of the technique. In particular, histological staining data can be overlaid and directly correlated with MALDI MS imaging data, providing the opportunity to highlight biochemical changes with changes in tissue morphology; a potentially powerful tool for the evaluation of drug safety. The final presentation of the day was from Philip Timmerman (Janssen, Belgium) who presented and commented on the European Bioanalysis Forum recommendations on tissue analysis which will be published in the forthcoming months in Bioanalysis.

\section{Quantitative technology}

Howard Hill (consultant, UK) began the second full day with a thought provoking talk on 'Healthcare industry - where to now?' covering the trends and challenges facing the industry. The UK healthcare budget is GBP $£ 140$ billion but declining, $50 \%$ of this budget is moving to community care, the US healthcare budget is $20 \%$ of GDP and unsustainable - can pharma continue to justify current margins on products? The number of new chemical entities is down, cost per molecule is up. Lifestyle changes and management could be cheaper than giving or discovering new drugs as the complexity of medication increases. Hill went on to explore the impact of relatively high labor costs in the pharma industry, attitudes of the next generation workforce and the pharma/CRO/academia triangle on drug development before rounding off the talk with a quizzical look at what the future might hold for the bioanalyst. This was followed by Mira Doig (ABS Laboratories, Hertfordshire, UK) who gave an overview of how bioanalysis has evolved over the last 40 years followed by Lloyd King (UCB, Slough, UK) on the versatility of HRMS platforms for both quantitative and qualitative data generation in research DMPK groups.
At the 2009 BioForum, David Perrett (Barts Medical School, London, UK) presented preliminary work on a detection system that can visualize residual protein on dental instruments after spraying with a fluorogenic reagent. In this follow up lecture, he reported how the system has been further developed both in terms of the chemistry and the digital imaging system; an instrument is now commercial available. $\mathrm{He}$ described some of the studies that had been carried out using different surgical instrument washers and detergents and the impact this has had on cleaning regimes in some hospitals. Studies on the level of protein contamination on single-use disposable instruments have led to warnings being issued by the MHRA. As ever, Perrett's talk was informative, entertaining and in places shocking with the take home message to avoid surgery if at all possible as you may get more than you bargained for. This session was completed with a talk by Essam Ghazaly (The London School of Medicine and Dentistry, London, UK) on the world of counterfeit drugs and was illustrated with some analytical case studies.

After a short break, the 'Quantitative Technology' theme was developed to cover large molecules and antidrug-antibody (ADA) bioanalysis. Louis Christodoulou spoke on the subject of 'ADA assessment: the advantages of transitioning to the electrochemical luminescence (ECL) platform'. This presentation focused on the advantages of using ECL immunoassays over traditional ELISA methodologies in the assessment of ADAs. The wider dynamic range, parallelism and sensitivity of the homogenous ECL immunoassay allow the measurement of ADA in the presence of drug PK (previously unachievable with the ELISA). The ability to generate ADA data in the presence of drug PK will allow a better understanding of immunogenicity, improve the evaluation of PK/PD data and inform the best dosing strategy. This was followed by Alex Hawes (Quotient Bio Analytical Sciences) who spoke on the subject of 'Challenges in keeping pace with immunogenicity advances throughout the clinical development of a biological therapeutic' and Mark Tatlock (Development and Discovery Solutions, UK) on 'Bioanalytical Tools for Characterization of Monoclonal Antibody Biosimilars'.

\section{Biomarker analysis}

After lunch the theme for session nine of the conference was biomarker analysis - peptide and proteins by mass spectrometry. Fabio Garofolo (Algorithm Pharma, Quebec, Canada), a familiar 
face at many international bioanalytical conferences, shared his approach to large molecule analysis using LC-MS instrumentation. He commented that HRMS opens new opportunities for quantification of peptides and proteins in biological samples during PK studies. The wide scan range of HRMS instruments is advantageous for intact peptide or protein quantification and these instruments are able to increase the selectivity while maintaining sensitivity. The presentation provided practical solutions for proteins and peptides quantification in a regulated environment with and without tryptic digestion by LC-MS. Jaap Wieling (QPS, The Netherlands) then spoke on 'Biomarkers in Alzheimer's - a variety of analytical techniques, new developments, challenges and validation approaches' before another short break.

On resumption, the biomarker theme moved towards the use in support of drug discovery. Lurence Meunier (Celerion, Switzerland) spoke on 'Application of new LC-MS technologies to peptide quantitation', which was followed by Neil Benson (Xenologiq, Kent, UK) on the topic of 'Generating insight from data using model based approaches', Barbara Bell (Covance, Leeds, UK) on 'Absolute quantitation of $4 \beta$-hydroxycholesterol in human plasma using a dual stable label LC-MS/MS approach with Atmospheric Pressure Chemical Ionisation' and Joe Palandra (Pfizer, USA) on 'Ultra-sensitive cytokine quantitation in tissues by immunoaffinity nano-LC-MS/MS', which wrapped up the day's proceeding.

\section{Regulations in bioanalysis}

The final morning of the conference saw a focus on regulations in bioanalysis: data integrity and assay validation. Earlier this year, the MHRA brought the first successful prosecution of a study director under the UK GLP Regulations following manipulation of bioanalytical data in a number of regulatory studies; Mr Eaton was sent to prison for 3 months [101]. Presenting for the first time publicly in the UK, Chris Gray of the MHRA opened the session by presenting on the issues identified in this investigation, their potential impact and how these and other data integrity issues can be identified and avoided.

Aptuit were first alerted to the regulatory anomalies when they identified a trend that method validation issues were all being resolved by a single analyst. Aptuit notified the MHRA that irregularities had been identified in analytical data and an inspection by the agency then lead to a full investigation involving the review of data from 650 different studies. Chromatography data had been manipulated such that batches that should have failed acceptance criteria appeared to pass. Manipulation of the data included: insertion of washes to mask carryover, re-injection of failed QC samples (and re-insertion of this data file into the batch sequence), multiple injection of whole batches, carry over assessment made after the low standard and miss integration of chromatographic peaks. Evidence for this was supported when acquisition times and dates for data files were reviewed along with file names and the presence of re-injections of the same batch in the raw data. Data had been manipulated in this fashion since 2003 and poor practices or breaches of the regulations were identified with half the analysts in the department.

As a result of this investigation, compliance was withdrawn from the analytical phase of approximately 300 studies. There was a potential impact to patients participating in clinical trials and preclinical studies had to be repeated; with both cost and additional animal use incurred. Ultimately, there was a delay in bringing several new therapies to market with an unquantifiable impact on human health. The study director/ principal investigator must make a statement of compliance for each regulated study/phase. The statement indicates whether or not the study was performed in accordance with the principles of GXP. Knowingly making a false statement is a criminal offence; hence Mr Eaton's subsequent prosecution and jail sentence.

The rest of the session before the mid-morning break was made up of Marcus Benton (QA consultant, UK) speaking on 'Readiness for a GXP inspection' - a practical guide, Karianna Mitchell (HLS, UK) with 'How to make a cross validation happy - a case study', and Garofolo on 'Importance of performing Incurred Sample Stability (ISS) for having a rugged and accurate omega-3 bioanalytical method - an illustrative case study on an industry hot topic'. After the short break, Peter van Amsterdam (Abbott, The Netherlands and Global Bioanalysis Consortium) compared and contrasted the various regulatory guidance requirements for method validation and sample analysis in a talk titled 'Global regulatory issues: one BA method, one validation, one report' [3]. This was followed by two talks on the subject of microsampling from Sangster and Kay Sommerville (both CRL, Rochdale, UK); a clear and concise explanation of how to use the capillary tube kits and the challenges of validating methods to support this type of sample collection. The final 
